Check for updates

Cite this: Mater. Chem. Front. 2020, 4, 862

Received 4th December 2019,

Accepted 6th January 2020

DOI: 10.1039/c9qm00733d

rsc.li/frontiers-materials

\title{
Engineering of a zero cross-talk fluorescent polymer nanoprobe for self-referenced ratiometric imaging of lysosomal hypochlorous acid in living cells†
}

\author{
Hongqing Wei, ${ }^{a}$ Rongjin Zeng, ${ }^{\star a}$ Shenglan Wang, ${ }^{a}$ Chong-Hua Zhang, ${ }^{a}$ Shu Chen, ${ }^{a}$ \\ Peisheng Zhang (D) *ab and Jian Chen (D) *ac
}

\begin{abstract}
The rational design of a zero cross-talk self-referenced ratiometric fluorescent probe for accurate molecular imaging in living cells is still a major challenge. Herein, we report a zero cross-talk fluorescent polymer nanoprobe (named ZC-FPN) with a self-referenced ratiometric effect for lysosomal hypochlorous acid $(\mathrm{HClO})$ imaging using single-wavelength excitation. The newly designed polymer nanoprobe (ZC-FPN) is prepared by using a simple co-precipitation method through self-assembly of two amphiphilic block copolymers, which are covalently linked with a porphyrin fluorophore ( $\mathrm{HClO}$-responsive unit) and a naphthalimide fluorophore (reference unit), respectively. Remarkably, the ZC-FPN displays an unprecedented separation of two emission peaks ( 191 nm), which can avoid the spectral cross-talk problem of the emission-shift type ratiometric probes and is highly favorable for high-resolution bioimaging. In addition, the as-prepared ZC-FPN also shows good water dispersibility, high selectivity, excellent long-term fluorescence stability (>10 weeks) and low cytotoxicity. More importantly, the positive charge of the amino moiety enhances the efficient uptake of the nanoprobe by cancer cells and enables the nanoprobe to selectively accumulate in lysosomes, achieving endogenous $\mathrm{HClO}$ imaging in living cells through fluorescence ratiometric imaging.
\end{abstract}

\section{Introduction}

Fluorescent probes integrated with an optical imaging technique have played vital roles in studying biological events owing to several unique features. ${ }^{1-19}$ Among the various strategies to construct fluorescent probes, polymer nanoparticle-based fluorescent nanoprobes (named polymer nanoprobes) have aroused great attention in many fields due to their intrinsic optical properties. ${ }^{20-29}$ To date, a series of currently developed polymer nanoprobes are

\footnotetext{
${ }^{a}$ Key Laboratory of Theoretical Organic Chemistry and Functional Molecule of Ministry of Education, Hunan Provincial Key Laboratory of Controllable Preparation and Functional Application of Fine Polymers, Hunan Province College Key Laboratory of QSAR/QSPR, Hunan Provincial Key Lab of Advanced Materials for New Energy Storage and Conversion, School of Chemistry and Chemical Engineering, Hunan University of Science and Technology, Xiangtan, Hunan 411201, China.E-mail: zrjxh2@126.com, pshzhang07@gmail.com, cj0066@gmail.com

${ }^{b}$ State Key Laboratory of Chemo/Biosensing and Chemometrics, Hunan University, Changsha 410082, P. R. China

${ }^{c}$ State Key Laboratory of Luminescent Materials and Devices, Key Laboratory of Luminescence from Molecular Aggregates of Guangdong Province,

South China University of Technology, Guangzhou 510640, P. R. China

$\dagger$ Electronic supplementary information (ESI) available: Synthesis procedures, ${ }^{1} \mathrm{H}$ NMR spectra, ${ }^{13} \mathrm{C}$ NMR, MS, absorption spectrum, fluorescence spectra, detection limit, cell viability, etc. See DOI: 10.1039/c9qm00733d
}

primarily based on either "one-emission signal" or "dual-emission signals (ratiometric)". ${ }^{30-35}$ Compared to the one-emission signalbased polymer nanoprobes, dual-emission signal-based polymer nanoprobes can enable more accurate detection and imaging through self-calibrating dual emission signals, thus providing more reliable quantitative analysis results. ${ }^{35}$

Generally, the commonly adopted strategies for designing ratiometric polymer nanoprobes are mainly dependent on either Intramolecular Charge Transfer (ICT) or Fluorescence Resonance Energy Transfer (FRET) mechanisms. ${ }^{36-43}$ Unfortunately, most of the ICT or FRET-based ratiometric polymer nanoprobes often show serious cross-talk between two emission bands, resulting in a low-contrast resolution performance for bioimaging. ${ }^{44}$ Therefore, the rational design of novel ratiometric fluorescent polymer nanoprobes with zero cross-talk of two emission signals for high-resolution imaging is still urgently demanded.

Porphyrin and its derivatives have been widely utilized for fluorescent probes due to their excellent features of high photostability, large Stokes shift $(>100 \mathrm{~nm})$, near-infrared (NIR) emission (>650 nm), etc. ${ }^{45-47}$ More importantly, our major new finding is that the NIR fluorescence of 4 -(10,15,20-triphenylporphyrin-5-yl)phenol (TPP-OH) derivatives can be sensitively and selectively quenched when exposed to hypochlorous acid ( $\mathrm{HClO})$ in 
an acid (pH 5.0) environment, making TPP-OH suitable for visualizing $\mathrm{HClO}$ in living cells (especially for lysosomal $\mathrm{HClO}$ ). On the other hand, naphthalimide and its derivatives have also been demonstrated to be promising probes for bioimaging because they possess high quantum yield, photostability and so on. ${ }^{48-50}$ Previously, Li et al. developed a naphthalimide-porphyrin based ratiometric fluorescence probe for imaging intracellular $\mathrm{Hg}^{2+}$ with a fixed excitation wavelength $(415 \mathrm{~nm}) .{ }^{51}$ This probe displays two well-resolved (zero cross-talk) emission spectra ( $\sim 125 \mathrm{~nm}$ ), which is beneficial for intracellular imaging with high resolution. Inspired by the work of $\mathrm{Li}$ and coworkers, we assume that both porphyrins and naphthalimides can be utilized to construct a zero cross-talk ratiometric fluorescent probe with single-wavelength excitation.

Polymeric micelles self-assembled by amphiphilic diblock copolymers have gained popularity as carriers for the fabrication of multifunctional fluorescent nanoprobes because of their unique advantages including tunable structure design, good water dispersibility, high photostability and excellent biocompatibility, etc. $^{52}$ In particular, the covalent incorporation of large $\pi-\pi$ conjugate chromophores (eg. porphyrins and naphthalimides) into the micelle core can not only effectively avoid the leakage problem of small dyes physically encapsulated in the micelle core, but also largely improve their brightness. ${ }^{44}$

In view of the good performance of TPP-OH for sensitive and selective HClO detection, we herein design a zero cross-talk fluorescent polymer nanoprobe (named ZC-FPN) with a selfreferenced ratiometric detection effect, obtained by self-assembling two fluorophore-contained amphiphilic block copolymers through a simple co-precipitation method (Scheme 1). The as-prepared ZC-FPN displays a single excitation wavelength $(405 \mathrm{~nm})$ and two well-resolved emission properties $(\sim 191 \mathrm{~nm})$, one from the HClO-responsive fluorophore (TPP-OH) and the other from the reference fluorophore (naphthalimide). Furthermore, the nanoprobe with a positive charge can especially stain lysosomes and further be used to detect endogenous $\mathrm{HClO}$ in living cells through fluorescence ratiometric imaging.

a)

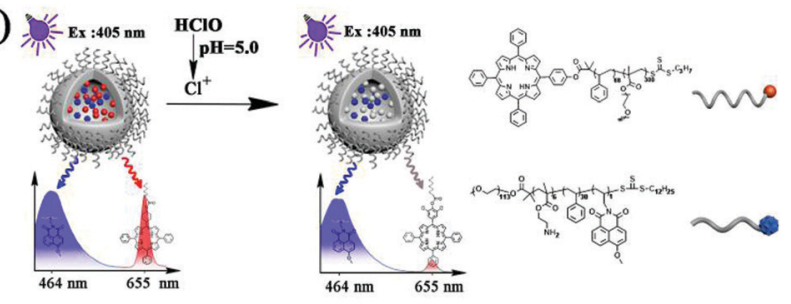

b)

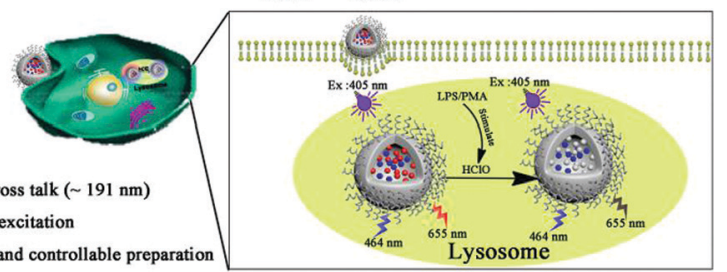

Scheme 1 Schematic illustration of ZC-FPN for self-referenced ratiometric detection of $\mathrm{HClO}$ (a) and ratiometric monitoring of endogenous $\mathrm{HClO}$ in lysosomes of living cells (b).

\section{Experimental section}

\section{Materials and instruments}

The relevant chemical reagents were obtained from commercial companies. The detailed synthesis, characterization, instruments, cytotoxicity and living cell imaging are given in the ESI. $\dagger$

\section{Results and discussion}

\section{Synthesis and characterization of ZC-FPN}

The selection of dyes was the key point for the fabrication of a zero cross-talk ratiometric fluorescent nanoprobe. The NIR dye (TPP-OH) served as the response unit for $\mathrm{HClO}$ recognition as HClO can induce the quenched fluorescence of TPP-OH, while naphthalimide was utilized as the internal reference unit based on its good stability when exposed to HClO. Significantly, the two dyes displayed a single-wavelength excitation (405 $\mathrm{nm}$ ) and two well-resolved emission peaks at 464 and $655 \mathrm{~nm}$, thereby enhancing the detection accuracy.

Next, two amphiphilic diblock copolymers (TPP- $b-\mathrm{PS}_{68}-b$ PEGMA $_{300}$ and $\left.\mathrm{PEO}_{113}-b-\mathrm{P}\left(\mathrm{AEMH}_{6}-c o-\mathrm{EANI}_{1}-\mathrm{co}-\mathrm{PS}_{30}\right)\right)$ containing TPP-OH and naphthalimide were synthesized by Reversible Addition-Fragmentation Chain Transfer Polymerization (Schemes S1 and S2, ESI $\dagger$ ). The relative intermediate and target compounds were identified by ${ }^{1} \mathrm{H} \mathrm{NMR},{ }^{13} \mathrm{C}$ NMR, Mass spectrum, Infrared (IR) spectrum and Gel Permeation Chromatograph (Fig. S1-S9 and Tables S1, S2, ESI $\dagger$ ).

Finally, by using a simple co-precipitation method, the above two amphiphilic diblock copolymers can be easily selfassembled together into a stable polymeric micelle (ZC-FPN) with an average particle size of about $22 \mathrm{~nm}$, which was measured by Dynamic Light Scattering (DLS, Fig. 1A). In addition, the spherical morphology of ZC-FPN with the corresponding average diameter was further analysed by Transmission Electron Microscopy (TEM, Fig. 1B). Remarkably, no obvious changes of diameter of ZC-FPN were visualized even after being stored at $25{ }^{\circ} \mathrm{C}$ for more than 30 days (Fig. 1C), and clear and stable aqueous dispersions of ZC-FPN can also be observed (Fig. 1D). The above results confirmed the excellent colloidal stability of ZC-FPN.

\section{Spectral response of ZC-FPN towards HClO}

With the ZC-FPN in hand, the HClO response of ZC-FPN $\left(48 \mu \mathrm{g} \mathrm{mL}^{-1}\right)$ was firstly investigated in a $\mathrm{pH}=5.0$ simulated physiological environment (PBS). The addition of $\mathrm{HClO}$ can result in an obvious decrease in absorption at $418 \mathrm{~nm}$ of ZC-FPN, indicating an efficient reaction between the TPP molecules and HClO (Fig. S10, ESI $\dagger$ ). Different from TPP molecules, naphthalimide molecules (EANI) were inert to $\mathrm{HClO}$, and thus the absorbance at $380 \mathrm{~nm}$ of ZC-FPN displayed no distinct change. As can be seen from the normalized excitation spectra of EANI and TPP-OH (Fig. S11, ESI $\dagger$ ), they share a common excitation wavelength at $405 \mathrm{~nm}$. Upon excitation at $405 \mathrm{~nm}$, a significantly decreased fluorescence (at $655 \mathrm{~nm}$ ) of TPP-OH can be observed in the presence of $\mathrm{HClO}$, while the fluorescence (at $464 \mathrm{~nm}$ ) of EANI exhibited no appreciable distinct change when exposed to $\mathrm{HClO}$ (Fig. S12, ESI $\dagger$ ). Significantly, the two well-resolved emission peaks 

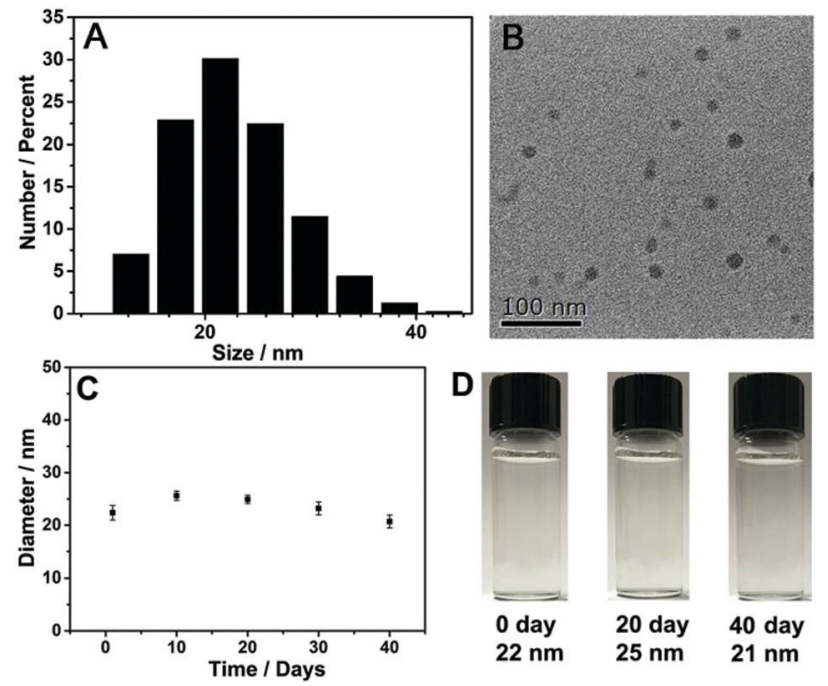

$22 \mathrm{~nm}$

$25 \mathrm{~nm}$

$21 \mathrm{~nm}$

Fig. 1 (A) Average diameter of ZC-FPN; (B) TEM of ZC-FPN; (C) average particle sizes and (D) images of ZC-FPN (1.44 $\left.\mathrm{mg} \mathrm{mL}^{-1}\right)$ with increasing time.

( $\sim 191 \mathrm{~nm})$ with a zero cross-talk property suggested an improved resolution for intracellular ratiometric imaging.

Subsequently, the capability of ZC-FPN for self-referenced ratiometric detection of $\mathrm{HClO}$ was carried out, as shown in Fig. 2A. When incubated with different concentrations of HClO, the ZC-FPN displayed gradually quenched fluorescence at $655 \mathrm{~nm}$, while the reference blue band at $464 \mathrm{~nm}$ remained almost unchanged. Notably, the quenched fluorescence of ZC-FPN at $655 \mathrm{~nm}$ may be ascribed to the intramolecular heavy atom effect of a chlorinated porphyrin structure (Scheme S3, ESI $\dagger$ ). ${ }^{53-55}$ Both the ${ }^{1} \mathrm{H}$ NMR (Fig. S13, ESI $\dagger$ ) and mass spectrum (Fig. S14, ESI $\dagger$ ) also confirmed this response mechanism. In addition, the fluorescence intensity ratios $\left(I_{464} / I_{655}\right)$ of ZC-FPN illustrated a good linear relationship with increasing $\mathrm{HClO}$ concentration (Fig. 2B), and the detection limit was determined to be $1.99 \mu \mathrm{M}$. The time response of ZC-FPN towards HClO was also investigated (Fig. S15, ESI $\dagger$ ). When exposed to HClO, the $I_{464} / I_{655}$ ratio of ZC-FPN increased remarkably and then reached a plateau in $7 \mathrm{~min}$. Therefore, $7 \mathrm{~min}$ was selected as a suitable time for sensing HClO.

Additionally, the selectivity experiments of ZC-FPN were carried out in the presence of various analytes commonly existing in biological matrices, and the results are given in Fig. 2C. Fortunately, only HClO could trigger a remarkable fluorescence intensity ratio change, while other potential interfering substances exhibited no perceptible effect, suggesting a good selectivity of ZC-FPN to HClO. Moreover, the pH influence of the fluorescence intensity ratio $\left(I_{464} / I_{655}\right)$ of ZC-FPN treated with and without $\mathrm{HClO}$ was also visualized. As shown in Fig. 2D, the $I_{464} / I_{655}$ ratio of ZC-FPN itself was low and unchanged obviously from $\mathrm{pH} 3.0$ to 9.0, while a remarkably enhanced $I_{464} / I_{655}$ ratio of ZC-FPN treated with $\mathrm{HClO}$ can be visualized from $\mathrm{pH} 3.0$ to 8.0, suggesting the good capability of ZC-FPN for the ratiometric detection of HClO in complex biosystems. Moreover, the preferable photostability and excellent long-term
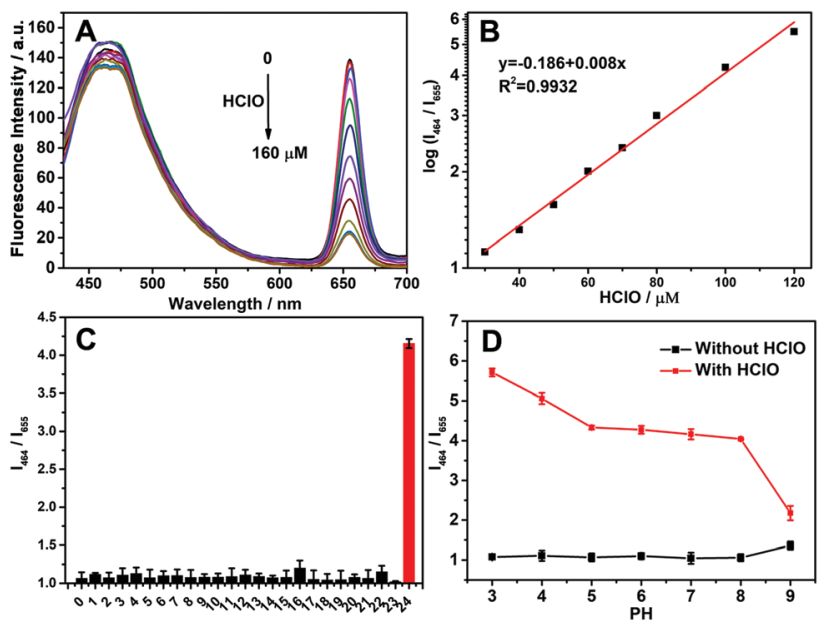

Fig. 2 (A) Ratiometric emission spectra of ZC-FPN $\left(48 \mu \mathrm{g} \mathrm{m}^{-1}\right)$ in the presence of $\mathrm{HClO}(0-160 \mu \mathrm{M})$ in $\mathrm{pH} 5.0$ PBS buffer solution $\left(\lambda_{\mathrm{ex}}=405 \mathrm{~nm}\right)$; (B) working curve of ZC-FPN to $\mathrm{HClO}(30-120 \mu \mathrm{M})$; (C) the fluorescence intensity ratio $\left(I_{464} / I_{655}\right)$ of ZC-FPN $\left(48 \mu \mathrm{g} \mathrm{mL}^{-1}\right)$ in the presence of various analytes (100 $\mu \mathrm{M}$ for $\mathrm{HClO}$ and $1 \mathrm{mM}$ for others): (0) blank, (1) $\mathrm{Na}^{+}$, (2) $\mathrm{Mg}^{2+}$, (3) $\mathrm{Ni}^{2+}$, (4) $\mathrm{Ca}^{2+}$, (5) $\mathrm{K}^{+}$, (6) $\mathrm{Zn}^{2+}$, (7) $\mathrm{Cu}^{2+}$, (8) $\mathrm{PO}_{4}{ }^{3+}$, (9) $\mathrm{HPO}_{4}{ }^{2+}$, (10) $\mathrm{H}_{2} \mathrm{PO}_{4}^{-}$, (11) $\mathrm{SO}_{4}{ }^{2+}$, (12) $\mathrm{NO}_{3}^{-}$, (13) $\mathrm{SO}_{3}^{2+}$, (14) $\mathrm{Cl}^{-}$, (15) $\mathrm{HS}^{-}$, (16) $\mathrm{H}_{2} \mathrm{O}_{2}$, (17) Cys, (18) GSH, (19) $\mathrm{Hcy},(20){ }^{1} \mathrm{O}_{2}$, (21) $\mathrm{Bu}-\mathrm{OOH},(22) \cdot \mathrm{OH}$, (23) $t$ - $\mathrm{BuO}{ }^{\bullet}$, (24) $\mathrm{ONOO}^{-}$, (25) $\mathrm{HClO}$; (D) effects of $\mathrm{pH}$ on the fluorescence intensity ratio $\left(/_{464} / /_{655}\right)$ for ZC-FPN $\left(48 \mu \mathrm{g} \mathrm{mL}^{-1}\right)$ without (black curve) and with (red curve) $\mathrm{HClO}(100 \mu \mathrm{M})$. Values are mean \pm s.d. for $n=5$.

fluorescence stability can make ZC-FPN more suitable for further intracellular imaging applications (Fig. S16 and S17, ESI†).

\section{Ratiometric imaging in living cells}

In view of the above good results, we further intend to evaluate the performance of ZC-FPN for exogenous/endogenous HClO imaging in lysosomes. Before imaging, the degree of toxicity of ZC-FPN toward the representative HeLa cells was evaluated. As can be seen from Fig. S18 (ESI $\dagger$ ), minimal cytotoxicity was visualized even at high concentrations $\left(100 \mu \mathrm{g} \mathrm{mL} \mathrm{m}^{-1}\right)$, indicating the excellent biocompatibility of ZC-FPN.

Some references suggested that nanoprobes with a positive charge can selectively localize to the lysosomes (Fig. S19, ESI $\dagger$ ). ${ }^{53,56}$ The co-localization experiments were carried out by using ZC-FPN and a lysosomal marker (Lyso-Tracker Green) to confirm the location of ZC-FPN in lysosomes. As shown in Fig. 3, the blue fluorescence image of ZC-FPN merged well with that of LysoTracker Green, and the high Pearson's coefficient and overlap coefficient were 0.9727 and 0.9748 , suggesting that ZC-FPN mainly stained lysosomes.

Next, ZC-FPN was utilized to ratiometrically detect exogenous $\mathrm{HClO}$ in lysosomes of HeLa cells by using $\mathrm{NaClO}$ as the HClO donor (Fig. 4). The red fluorescence intensities gradually decreased with increasing $\mathrm{HClO}$ concentrations, while no obvious changes can be visualized from the blue channel. Correspondingly, the ratio images $\left(F_{\text {blue }} / F_{\text {red }}\right)$ also supported this variation tendency (Fig. 4M-P). In view of the good ability of ZC-FPN for exogenous HClO imaging, we then evaluated its performance for endogenous HClO detection in Raw264.7 macrophage cells by stimulating cells 


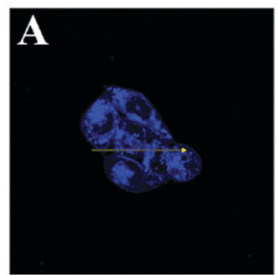

$D^{\text {lnas }}$

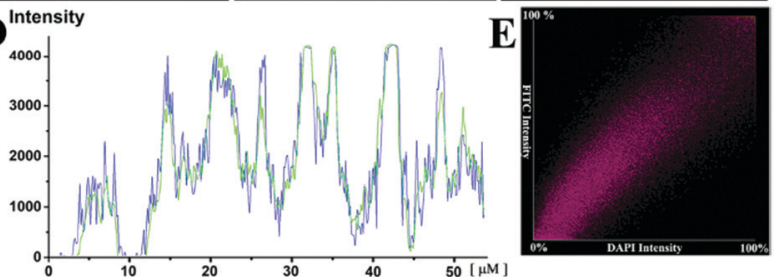

Fig. 3 Co-localization experiments of (A) ZC-FPN (48 $\left.\mu \mathrm{g} \mathrm{mL}^{-1}\right)$ and (B) Lyso-Tracker Green (500 nM) in HeLa cells. (C) Overlay of (A) and (B); (D) intensity correlation plot of ROI from (A) and (B); $(E)$ the correlation plot of (A) and (B). Blue channel: $\lambda_{\mathrm{ex}}=405 \mathrm{~nm}$ and $\lambda_{\mathrm{em} 1}=440-500 \mathrm{~nm}$; green channel: $\lambda_{\mathrm{ex}}=504 \mathrm{~nm}$ and $\lambda_{\mathrm{em}}=520-540 \mathrm{~nm}$ scale bar: $20 \mu \mathrm{m}$.

with Lipopolysaccharide (LPS) and Phorbol Myristate Acetate (PMA). ${ }^{57,58}$ As can be seen from Fig. 5D and G, there were strong fluorescence signals in both the blue and red channels, indicating the efficient uptake of ZC-FPN by RAW264.7 macrophage cells. In contrast, when the RAW264.7 cells were co-treated with LPS, PMA and ZC-FPN, the blue channel still displayed a bright fluorescence signal (Fig. 5E), while an obvious weak fluorescence signal in the red channel was noted (Fig. $5 \mathrm{H}$ ). To verify that the endogenously generated $\mathrm{HClO}$ were responsible for the intracellular fluorescence

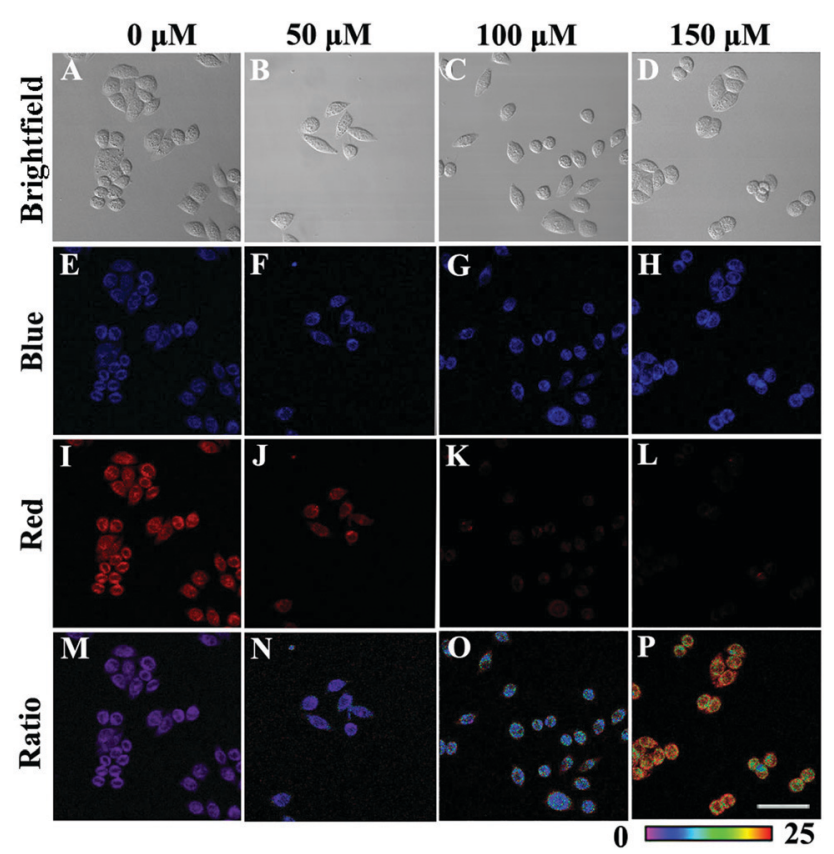

Fig. 4 Confocal fluorescence microscopy images of HeLa cells treated with ZC-FPN (48 $\mu \mathrm{g} \mathrm{mL} \mathrm{mL}^{-1}$ ) followed by incubation with 0 ( $\mathrm{A}, \mathrm{E}$ and $\mathrm{I}$ ), 50 (B, F and J), 100 (C, G and $\mathrm{K}$ ) and $150 \mu \mathrm{M}(\mathrm{D}, \mathrm{H}$ and $\mathrm{L}) \mathrm{HClO}$. (M, N, O and $P)$ The ratio images $\left(F_{\text {blue }} / F_{\text {red }}\right) . \lambda_{\text {ex }}=405 \mathrm{~nm}, \lambda_{\mathrm{em} 1}=440-500 \mathrm{~nm}$ (blue channel), and $\lambda_{\mathrm{em} 2}=640-670 \mathrm{~nm}$ (red channel) scale bar: $20 \mu \mathrm{m}$.

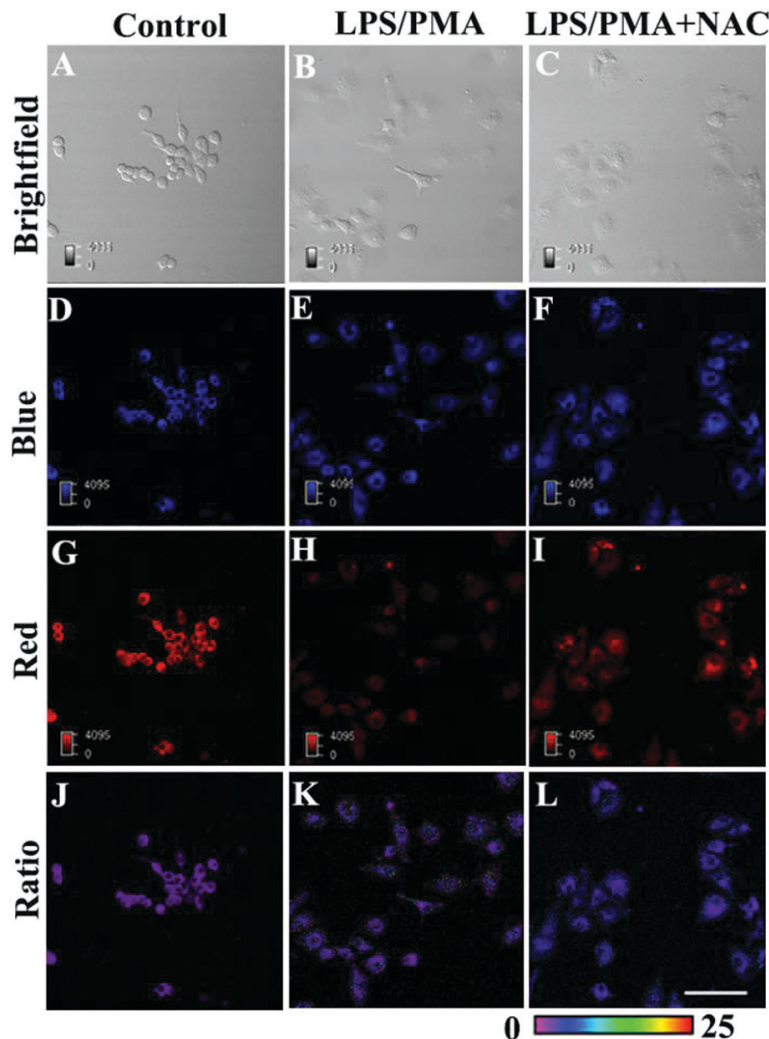

Fig. 5 Confocal fluorescence microscopy images of RAW264.7 macrophage cells. (A, D and G) Cells incubated with ZC-FPN (48 $\left.\mathrm{g} \mathrm{mL}^{-1}\right) ;(B, E$, and H) cells incubated with LPS ( $\left.1 \mathrm{mg} \mathrm{mL}^{-1}\right)$, followed by co-incubation with PMA $\left(1 \mathrm{mg} \mathrm{mL}^{-1}\right)$ and ZC-FPN (48 $\left.\mathrm{g} \mathrm{mL}^{-1}\right)$; (C, F, and I) cells incubated with LPS (1 $\mathrm{mg} \mathrm{mL}^{-1}$ ) and NAC (2 $\left.\mathrm{mM}\right)$, followed by co-incubation with PMA (1 $\mathrm{mg} \mathrm{mL}^{-1}$ ) and ZC-FPN (48 $\left.\mu \mathrm{g} \mathrm{mL}^{-1}\right)$; (J, K and L) the ratio images $\left(F_{\text {blue }} / F_{\text {red }}\right) ; \lambda_{\text {ex }}=405 \mathrm{~nm}, \lambda_{\mathrm{em} 1}=440-500 \mathrm{~nm}$ (blue channel), and $\lambda_{\mathrm{em} 2}=$ 640-670 nm (red channel) scale bar: $20 \mu \mathrm{m}$.

signal changes, a control experiment was performed by treating the LPS/PMA stimulated RAW264.7 cells with $N$-acetylcysteine (NAC, an effective ROS scavenger). ${ }^{59}$ Because NAC can efficiently remove the endogenously generated $\mathrm{HClO}$, and thus result in a very low $\mathrm{HClO}$ level in RAW264.7 cells. As expected, both the blue and red channels exhibited bright fluorescence signals (Fig. 5F and I), indicating that the quenched fluorescence signals of the red channels indeed originated from the endogenously produced HClO. Additionally, when the RAW264.7 cells were treated with NAC and ZC-FPN, both the blue and red channels exhibited strong fluorescence signals (Fig. S20, ESI $\dagger$ ), indicating that NAC cannot induce the fluorescence signal change of the red channel. These results revealed the good ability of ZC-FPN for exogenous/ endogenous HClO imaging in living cells.

\section{Conclusion}

To sum up, we have successfully developed a zero cross-talk fluorescent polymer nanoprobe (ZC-FPN) for HClO imaging in lysosomes with a ratiometric effect. The rationally designed ZC-FPN possesses some prominent advantages over the previously described $\mathrm{HClO}$ fluorescent probes with a dual-emission 
property (Table S1, ESI $\dagger$ ): First, the synthesized ZC-FPN can operate in a $100 \%$ aqueous solution, whereas the previously described $\mathrm{HClO}$ fluorescent probes operated in mixed organicaqueous solvent systems; second, the ZC-FPN illustrates an ideal single excitation wavelength and well-resolved dual emissions ( $191 \mathrm{~nm}$ ), which can avoid the spectral cross-talk issue of the emission-shift type or the FRET-based ratiometric probes and is highly beneficial for high-resolution bioimaging; third, benefiting from the positive charge of the amino moiety modified nanoparticles, the ZC-FPN with good cell-membrane permeability can selectively locate at lysosomes, and further be utilized to detect exogenous and endogenous $\mathrm{HClO}$ in the lysosomes of living cells by using fluorescence ratiometric imaging.

\section{Conflicts of interest}

The authors declare no competing financial interest.

\section{Acknowledgements}

The present work was financially supported by NSFC (Project no. 51873058, 51603067 and 51773056), Hunan Provincial Natural Science Foundation of China (No. 2019JJ60005 and 2018JJ3143), Scientific Research Fund of Hunan Provincial Education Department (Project no. 19B204), China Postdoctoral Science Foundation (2017M622571 and 2018T110824), Open Project Program of State Key Laboratory of Chemo/Biosensing and Chemometrics (Project no. 2016019 and 2018011), and the Open Fund of the State Key Laboratory of Luminescent Materials and Devices (South China University of Technology, 2019-skllmd-09).

\section{Notes and references}

1 S.-H. Park, N. Kwon, J.-H. Lee, J. Yoon and I. Shin, Synthetic ratiometric fluorescent probes for detection of ions, Chem. Soc. Rev., 2019, DOI: 10.1039/C9CS00243J.

2 M. Gao, F. Yu, C. Lv, J. Choo and L. Chen, Fluorescent chemical probes for accurate tumor diagnosis and targeting therapy, Chem. Soc. Rev., 2017, 46, 2237-2271.

3 Y. M. Poronik, K. V. Vygranenko, D. Gryko and D. T. Gryko, Rhodols-synthesis, photophysical properties and applications as fluorescent probes, Chem. Soc. Rev., 2019, 48, 5242-5265.

4 W. Sun, M. Li, J. Fan and X. Peng, Activity-based sensing and theranostic probes based on photoinduced electron transfer, Acc. Chem. Res., 2019, 52, 2818-2831.

5 J. Ohata, K. J. Bruemmer and C. J. Chang, Activity-based sensing methods for monitoring the reactive carbon species carbon monoxide and formaldehyde in living systems, Acc. Chem. Res., 2019, 52, 2841-2848.

6 D. Wang and B. Z. Tang, Aggregation-induced emission luminogens for activity-based sensing, Acc. Chem. Res., 2019, 52, 2559-2570.

7 S. Yang, C. C. Guo, Y. Li, J. R. Guo, J. Xiao, Z. H. Qing, J. S. Li and R. H. Yang, A ratiometric two-photon fluorescent cysteine probe with well-resolved dual emissions based on intramolecular charge transfer-mediated two-photon-FRET integration mechanism, ACS Sens., 2018, 3, 2415-2422.

8 P. Zhang, Y. Tian, H. Liu, J. Ren, H. Wang, R. Zeng, Y. Long and J. Chen, In vivo imaging of hepatocellular nitric oxide using a hepatocyte-targeting fluorescent sensor, Chem. Commun., 2018, 54, 7231-7234.

9 L. Wu, X. Li, Y. Ling, C. Huang and N. Jia, Morpholine derivative-functionalized carbon dots-based fluorescent probe for highly selective lysosomal imaging in living cells, ACS Appl. Mater. Interfaces, 2017, 9, 28222-28232.

10 C. Huang, T. Jia, M. Tang, Q. Yin, W. Zhu, C. Zhang, Y. Yang, N. Jia, Y. Xu and X. Qian, Selective and ratiometric fluorescent trapping and quantification of protein vicinal dithiols and in situ dynamic tracing in living cells, J. Am. Chem. Soc., 2014, 136, 14237-14244.

11 L. Wu, A. C. Sedgwick, X. Sun, S. D. Bull, X. P. He and T. D. James, Reaction-based fluorescent probes for the detection and imaging of reactive oxygen, nitrogen, and sulfur species, Acc. Chem. Res., 2019, 52, 2582-2597.

12 Y. Yue, F. Huo, F. Cheng, X. Zhu, T. Mafireyi, R. M. Strongin and C. Yin, Functional synthetic probes for selective targeting and multi-analyte detection and imaging, Chem. Soc. Rev., 2019, 48, 4155-4177.

13 D. Wu, L. Chen, Q. Xu, X. Chen and J. Yoon, Design principles, sensing mechanisms, and applications of highly specific fluorescent probes for $\mathrm{HOCl} / \mathrm{OCl}^{-}$, Acc. Chem. Res., 2019, 52, 2158-2168.

14 X. Wu, W. Shi, X. Li and H. Ma, Recognition moieties of small molecular fluorescent probes for bioimaging of enzymes, Acc. Chem. Res., 2019, 52, 1892-1904.

15 P. Gao, W. Pan, N. Li and B. Tang, Fluorescent probes for organelle-targeted bioactive species imaging, Chem. Sci., 2019, 10, 6035-6071.

16 J. L. Zhu, Z. Xu, Y. Yang and L. Xu, Small-molecule fluorescent probes for specific detection and imaging of chemical species inside lysosomes, Chem. Commun., 2019, 55, 6629-6671.

17 L. He, B. Dong, Y. Liu and W. Lin, Fluorescent chemosensors manipulated by dual/triple interplaying sensing mechanisms, Chem. Soc. Rev., 2016, 45, 6449-6461.

18 L. Wu, Y. Wang, T. D. James, N. Jia and C. Huang, A hemicyanine based ratiometric fluorescence probe for mapping lysosomal pH during heat stroke in living cells, Chem. Commun., 2018, 54, 5518-5521.

19 L. Wu, Q. Yang, L. Liu, A. C. Sedgwick, A. J. Cresswell, S. D. Bull, C. Huang and T. D. James, ESIPT-based fluorescence probe for the rapid detection of hypochlorite $\left(\mathrm{HOCl} / \mathrm{ClO}^{-}\right)$, Chem. Commun., 2018, 54, 8522-8525.

$20 \mathrm{~K}$. Li and B. Liu, Polymer-encapsulated organic nanoparticles for fluorescence and photoacoustic imaging, Chem. Soc. Rev., 2014, 43, 6570-6597.

21 J. He, C. Li, L. Ding, Y. Huang, X. Yin, J. Zhang, J. Zhang, C. Yao, M. Liang, R. P. Pirraco, J. Chen, Q. Lu, R. Baldridge, Y. Zhang, M. Wu, R. L. Reis and Y. Wang, Tumor targeting strategies of smart fluorescent nanoparticles and their applications in cancer diagnosis and treatment, Adv. Mater., 2019, 31, 1902409. 
$22 \mathrm{~J} . \mathrm{Li}$ and $\mathrm{K} . \mathrm{Pu}$, Development of organic semiconducting materials for deep-tissue optical imaging, phototherapy and photoactivation, Chem. Soc. Rev., 2019, 48, 38-71.

$23 \mathrm{X}$. Zhen and $\mathrm{K}$. Pu, Development of optical nanoprobes for molecular imaging of reactive oxygen and nitrogen species, Nano Res., 2018, 11, 5258-5280.

24 J. Yu, Y. Rong, C.-T. Kuo, X.-H. Zhou and D. T. Chiu, Recent advances in the development of highly luminescent semiconducting polymer dots and nanoparticles for biological imaging and medicine, Anal. Chem., 2016, 89, 42-56.

25 F. Lv, T. Qiu, L. Liu, J. Ying and S. Wang, Recent advances in conjugated polymer materials for disease diagnosis, Small, 2016, 12, 696-705.

26 L. Yan, Y. Zhang, B. Xu and W. Tian, Fluorescent nanoparticles based on AIE fluorogens for bioimaging, Nanoscale, 2016, 8, 2471-2487.

27 O. S. Wolfbeis, An overview of nanoparticles commonly used in fluorescent bioimaging, Chem. Soc. Rev., 2015, 44, 4743-4768.

$28 \mathrm{~J} . \mathrm{Hu}$ and S. Liu, Engineering responsive polymer building blocks with host-guest molecular recognition for functional applications, Acc. Chem. Res., 2014, 47, 2084-2095.

29 A. Reisch and A. S. Klymchenko, Fluorescent polymer nanoparticles based on dyes: seeking brighter tools for bioimaging, Small, 2016, 12, 1968-1992.

30 P. Zhang, X. Nie, M. Gao, F. Zeng, A. Qin, S. Wu and B. Z. Tang, A highly selective fluorescent nanoprobe based on AIE and ESIPT for imaging hydrogen sulfide in live cells and zebrafish, Mater. Chem. Front., 2017, 1, 838-845.

31 K. C. Chong, F. Hu and B. Liu, AIEgen bioconjugates for specific detection of disease-related protein biomarkers, Mater. Chem. Front., 2019, 3, 12-24.

32 Q. Wang, B. Xia, J. Xu, X. Niu, J. Cai, Q. Shen, W. Wang, W. Huang and Q. Fan, Biocompatible small organic molecule phototheranostics for NIR-II fluorescence/photoacoustic imaging and simultaneous photodynamic/photothermal combination therapy, Mater. Chem. Front., 2019, 3, 650-655.

33 P. Zhang, J. Chen, F. Huang, Z. Zeng, J. Hu, P. Yi, F. Zeng and $\mathrm{S}$. Wu, One-pot fabrication of polymer nanoparticle-based chemosensors for $\mathrm{Cu}^{2+}$ detection in aqueous media, Polym. Chem., 2013, 4, 2325-2332.

34 Y. Li, S. Yang, L. Guo, Y. Xiao, J. Q. Luo, Y. H. Li, M. S. Wong and R. H. Yang, Differentiation of intracellular hyaluronidase isoform by degradable nanoassembly coupled with RNA-binding fluorescence amplification, Anal. Chem., 2019, 91, 6887-6893.

35 X. Huang, J. Song, B. C. Yung, X. Huang, Y. Xiong and X. Chen, Ratiometric optical nanoprobes enable accurate molecular detection and imaging, Chem. Soc. Rev., 2018, 47, 2873-2920.

36 Y. Huang, P. Zhang, M. Gao, F. Zeng, A. Qin, S. Wu and B. Z. Tang, Ratiometric detection and imaging of endogenous hypochlorite in live cells and in vivo achieved by using an aggregation induced emission (AIE)-based nanoprobe, Chem. Commun., 2016, 52, 7288-7291.

37 J. Peng, X. Hou, F. Zeng and S. Wu, Fluorescent nanoprobe for in vivo ratiometric imaging of endogenous hydrogen peroxide resulted from drug-induced organ damages, Biosens. Bioelectron., 2017, 94, 278-285.

38 Y. Hong, H. Wang, M. Xue, P. Zhang, W. Liu, S. Chen, R. Zeng, J. Cui, Y. Gao and J. Chen, Rational design of ratiometric and lysosome-targetable AIE dots for imaging endogenous HClO in live cells, Mater. Chem. Front., 2019, 3, 203-208.

39 P. Zhang, H. Wang, Y. Hong, M. Yu, R. Zeng, Y. Long and J. Chen, Selective visualization of endogenous hypochlorous acid in zebrafish during lipopolysaccharide-induced acute liver injury using a polymer micelles-based ratiometric fluorescent probe, Biosens. Bioelectron., 2017, 99, 318-324.

40 G. Chen, F. Song, J. Wang, Z. Yang, S. Sun, J. Fan, X. Qiang, X. Wang, B. Dou and X. Peng, FRET spectral unmixing: a ratiometric fluorescent nanoprobe for hypochlorite, Chem. Commun., 2012, 48, 2949-2951.

41 J. Qiao, Z. Liu, Y. Tian, M. Wu and Z. Niu, Multifunctional self-assembled polymeric nanoprobes for FRET-based ratiometric detection of mitochondrial $\mathrm{H}_{2} \mathrm{O}_{2}$ in living cells, Chem. Commun., 2015, 51, 3641-3644.

42 L. Wu, I. C. Wu, C. C. DuFort, M. A. Carlson, X. Wu, L. Chen, C. T. Kuo, Y. Qin, J. Yu, S. R. Hingorani and D. T. Chiu, Photostable ratiometric pdot probe for in vitro and in vivo imaging of hypochlorous acid, J. Am. Chem. Soc., 2017, 139, 6911-6918.

43 Y. L. Chiu, S. A. Chen, J. H. Chen, K. J. Chen, H. L. Chen and H. W. Sung, A dual-emission forster resonance energy transfer nanoprobe for sensing/imaging $\mathrm{pH}$ changes in the biological environment, ACS Nano, 2010, 4, 7467-7474.

44 S. Wang, K. Gu, Z. Guo, C. Yan, T. Yang, Z. Chen, H. Tian and W. H. Zhu, Self-assembly of a monochromophore-based polymer enables unprecedented ratiometric tracing of hypoxia, Adv. Mater., 2018, 31, 1805735.

45 Y. Ding, Y. Tang, W. Zhu and Y. Xie, Fluorescent and colorimetric ion probes based on conjugated oligopyrroles, Chem. Soc. Rev., 2015, 44, 1101-1112.

46 Q. Zhang, X. Dong, K.-P. Wang, T.-T. Zhu, F.-N. Sun, S.-X. Meng and Y.-Q. Feng, Glycine-conjugated porphyrin fluorescent probe with iRGD for live cell imaging, Chin. Chem. Lett., 2017, 28, 777-781.

47 A. Khadria, J. Fleischhauer, I. Boczarow, J. D. Wilkinson, M. M. Kohl and H. L. Anderson, Porphyrin dyes for nonlinear optical imaging of live cells, iScience, 2018, 4, 153-163.

48 S. Banerjee, E. B. Veale, C. M. Phelan, S. A. Murphy, G. M. Tocci, L. J. Gillespie, D. O. Frimannsson, J. M. Kelly and T. Gunnlaugsson, Recent advances in the development of 1 , 8-naphthalimide based DNA targeting binders, anticancer and fluorescent cellular imaging agents, Chem. Soc. Rev., 2013, 42, 1601-1618.

49 Z. R. Dai, G. B. Ge, L. Feng, J. Ning, L. H. Hu, Q. Jin, D. D. Wang, X. Lv, T. Y. Dou, J. N. Cui and L. Yang, A highly selective ratiometric two-photon fluorescent probe for human cytochrome P450 1A, J. Am. Chem. Soc., 2015, 137, 14488-14495.

50 Z. Xie, M. Fu, B. Yin and Q. Zhu, Research progress in 1,8naphthalimide-based fluorescent probes for two-photon imaging, J. Org. Chem., 2018, 38, 1364. 
51 C. Y. Li, X. B. Zhang, L. Qiao, Y. Zhao, C. M. He, S. Y. Huan, L. M. Lu, L. X. Jian, G. L. Shen and R. Q. Yu, Naphthalimide-porphyrin hybrid based ratiometric bioimaging probe for $\mathrm{Hg}^{2+}$ : well-resolved emission spectra and unique specificity, Anal. Chem., 2009, 81, 9993-10001.

52 C. Zhao, X. Zhang, K. Li, S. Zhu, Z. Guo, L. Zhang, F. Wang, Q. Fei, S. Luo, P. Shi, H. Tian and W.-H. Zhu, Förster resonance energy transfer switchable self-assembled micellar nanoprobe: ratiometric fluorescent trapping of endogenous $\mathrm{H}_{2} \mathrm{~S}$ generation via fluvastatin-stimulated upregulation, J. Am. Chem. Soc., 2015, 137, 8490-8498.

53 H. Wang, P. Zhang, Y. Hong, B. Zhao, P. Yi and J. Chen, Ratiometric imaging of lysosomal hypochlorous acid enabled by FRET-based polymer dots, Polym. Chem., 2017, 8, 5795-5802.

54 M. Xue, H. Wang, J. Chen, J. Ren, S. Chen, H. Yang, R. Zeng, Y. Long and P. Zhang, Ratiometric fluorescent sensing of endogenous hypochlorous acid in lysosomes using AIE-based polymeric nanoprobe, Sens. Actuators, B, 2019, 282, 1-8.

55 J. Ren, P. Zhang, H. Liu, C. Zhang, Y. Gao, J. Cui and J. Chen, Single-dye-doped fluorescent nanoprobe enables self-referenced ratiometric imaging of hypochlorous acid in lysosomes, Sens. Actuators, B, 2020, 304, 127299.

56 J. Chen, Y. Tang, H. Wang, P. Zhang, Y. Li and J. Jiang, Design and fabrication of fluorescence resonance energy transfer-mediated fluorescent polymer nanoparticles for ratiometric sensing of lysosomal $\mathrm{pH}, \mathrm{J}$. Colloid Interface Sci., 2016, 484, 298-307.

57 Y. Koide, Y. Urano, K. Hanaoka, T. Terai and T. Nagano, Development of an Si-rhodamine-based far-red to nearinfrared fluorescence probe selective for hypochlorous acid and its applications for biological imaging, J. Am. Chem. Soc., 2011, 133, 5680-5682.

58 W. Zhang, W. Liu, P. Li, J. Kang, J. Wang, H. Wang and B. Tang, Reversible two-photon fluorescent probe for imaging of hypochlorous acid in live cells and in vivo, Chem. Commun., 2015, 51, 10150-10153.

59 Y. W. Jun, S. Sarkar, S. Singha, Y. J. Reo, H. R. Kim, J. Kim, Y. Chang and K. H. Ahn, A two-photon fluorescent probe for ratiometric imaging of endogenous hypochlorous acid in live cells and tissues, Chem. Commun., 2017, 53, 10800-10803. 